\title{
In-situ observation of ULF wave activities associated with substorm expansion phase onset and current disruption
}

\author{
J. Liang ${ }^{1,2}$, W. W. Liu ${ }^{1}$, E. F. Donovan ${ }^{2}$, and E. Spanswick ${ }^{2}$ \\ ${ }^{1}$ Space Science Branch, Canadian Space Agency, St-Hubert, Quebec, Canada \\ ${ }^{2}$ Department of Physics and Astronomy, University of Calgary, Calgary, Alberta, Canada \\ Received: 15 October 2008 - Revised: 8 April 2009 - Accepted: 8 May 2009 - Published: 13 May 2009
}

\begin{abstract}
In this paper we present two substorm events with coordinated ground-based and in-situ THEMIS observations, and focus our interest on the wave activities in Pi1 and $\mathrm{Pi} 2$ bands from minutes before the substorm expansion phase (EP) onset to minutes after the local current disruption (CD). We find that Pi2 band (40-100s) wave appears 1-2 min before the substorm onset and last over the entire EP interval, while higher-frequency wave within Pi1 band (10-30 s) emerges within few tens of seconds after the EP onset, intensifies during the local $\mathrm{CD}$, and fades afterwards. The pre-onset $\mathrm{Pi} 2$ waves are attributed to a ballooning mode which acts as the seed perturbation to the substorm EP onset process. The azimuthal wavenumber estimated from the Doppler shift nature of the ballooning mode is consistent with the longitudinal "wavelength" inferred from the onset auroral structures. The Pil waves appearing within few tens of seconds after the EP onset are interpreted as supportive of a two-fluid instability mode of thin current sheet investigated in an accompanying paper (Liu and Liang, 2009). During the local CD, broadband wave activities from Pi2 band to well above the ion gyrofrequency are observed, suggesting the coexistence of various plasma instabilities featuring different frequency ranges.
\end{abstract}

Keywords. Magnetospheric physics (Auroral phenomena; Plasma waves and instabilities; Storms and substorms)

\section{Introduction}

Ultra-low frequency (ULF) waves have long been recognized as an essential element in magnetospheric physics. On global scales the waves provide a key link between different magnetospheric/ionospheric regions; on local scale the

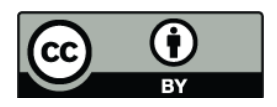

Correspondence to: J. Liang

(jliang@phys.ucalgary.ca) waves are typically representative of the plasma instability modes responsible for transient magnetospheric reconfigurations, among which the substorm expansion phase (EP) onset and the accompanying current disruption (CD) process are outstanding examples. Magnetic field fluctuations with wide frequency range during substorm intervals have been investigated by many researchers based upon different spacecraft measurements and analysis approaches (Holter et al., 1995; Lui and Najmi, 1997; Sigsbee et al., 2002; Shiokawa et al., 2005; Saito et al., 2008a, b; Lui et al., 2008). As a methodological preparations as well as noteworthy predecessors to this study we refer the readers to Holter et al. (1995) and Saito et al. (2008a, b) for an example of the use of wavelet analysis on a time-frequency decomposition of ULF wave components, and the efforts to discern observed wave components in terms of known plasma modes. The electric/magnetic field oscillations with period $45-65 \mathrm{~s}$ observed during the most active period of a substorm EP are interpreted as standing Alfvénic modes trapped in an equatorial current layer (Holter et al., 1995). On the other hand, one specific type of $\mathrm{Pi} 2$ waves at $\sim 60$ s period emerging $1-3 \mathrm{~min}$ prior to the EP onset was suggested by Saito et al. (2008b) as a manifestation of ballooning mode which was dubbed by many as the candidate instability leading to substorm EP onset (Pu et al., 1997; Erickson et al., 2000; Cheng, 2004). The other major classes of the onset-related instability, the crossfield current instabilities (CCIs) (Lui et al., 1991) are kinetic in nature and characterized with frequency range between the ion gyrofrequency and the lower hybrid frequency; therefore in a typical thin current sheet configuration the CCI wave band is largely above the Pi1 range. Arnoldy et al. (1998) investigated the Pil band waves/pulsations from space and ground observations; in many events they identified an abrupt intensification of Pil waves from GOES measurements in conjunction with the start of the local dipolarization. In an event study Lessard et al. (2006) found that the observed Pi1 waves at GOES are compressional in nature and thus

Published by Copernicus Publications on behalf of the European Geosciences Union. 
presumably a fast mode. The existence of Pil wave activities accompanying the local CD process were also revealed in many other above-cited studies, though their theoretical interpretation remains somewhat lacking so far.

While the above studies unambiguously revealed the abundance of ULF wave activities at Pi1/Pi2 bands in a substorm onset process, some deficiencies in their experimental approaches prevented the clarification of the characteristic wave modes in more detail. For example, (a) in many of the above studies there is none or only low-resolution global auroral observations such that the substorm EP onset, which is a key epoch in the entire disturbance, could not be properly determined with requisite precision. It is common that the local CD recorded by a single satellite, contingent upon its location with respect to the place of onset, lagged the initial EP onset by several minutes; such time delay can significantly affect the interpretation of the observed wave mode, i.e., locally excited or remotely triggered through propagation; (b) There is no spatial and propagational information of the wave based solely upon single-satellite observations, limiting the efforts to characterize the wave mode and compare with theoretical expectations (e.g., viz the dispersion relationship). Saito et al. (2008a) proposed a MHD fitting method to estimate the propagational information based upon single-satellite measurements, which only works for frequency much lower than the ion gyrofrequency (valid for Pi2 band but questionable for Pi1), and with assumption of slow and incompressible ambient flows, which is likely violated during substorm EP intervals.

Multi-satellite observations of ULF waves were implemented successfully in the Cluster mission (e.g., see Volwerk, 2006). There is little doubt that the NASA's Time History of Events and Macroscale Interactions during Substorms (THEMIS) mission ushered in a new era of substorm studies. While the primary objective of THEMIS mission is a timing between the mid-tail disturbance (e.g., fast flows) and the near-Earth disturbance (e.g., CD) in a substorm sequence, THEMIS has another remarkable feature in that it provides an unprecedented opportunity of coordinated in-situ and ground-based observations of the seconds-scale dynamics of substorm process, and thus is particularly suited for the study of ULF wave activity. In general, satellite observations may yield the temporal characteristic such as wave frequency and growth rate (in terms of instability), while ground-based auroral observations with 3-s cadence not only provide the most accurate way of EP onset timing to establish a reference epoch for the wave activity, but also have unmatched capability to provide the spatial structures, such as the longitudinal wavelength, of substorm intensifications (Liang et al., 2008). Global ASIs and multi-satellite conjunctions also complement each other to yield useful propagational information of the substorm disturbances (Donovan et al., 2008).

As an illustration of the above-advocated research strategy, we will present in this paper two substorm events with coordinated in-situ and ground-based THEMIS observations to investigate the ULF wave activities during substorm EP onset and local CD. We then discuss the nature of the observed wave modes with different time-frequency characteristics and their possible interaction with the local current sheet. In particular, the Pi2 band (40-100s) waves $\sim 1 \mathrm{~min}$ preceding substorm EP onset is attributed to the ballooning mode (Cheng, 2004; Saito et al., 2008b), while the Pi1 band (10-30 s) waves intensifying in close conjunction with EP onset and local CD are consistent with the theoretical prediction of a two-fluid MHD instability mode of thin current sheet recently studied by Liu and Liang (2009).

\section{THEMIS observations}

The main instruments used in this study are the THEMIS ground-based all-sky imager (ASI, see Mende et al., 2008 for locations and instrumental details) and the Fluxgate Magnetometer (FGM, see Auster et al., 2008) as well as the Electrostatic Analyzer (ESA, see McFadden et al., 2008) onboard of the probes. Before delving into observations we briefly explain two key epochs repeatedly mentioned in this paper, the substorm EP onset and the local CD onset. The EP onset (also known as "auroral" onset) is determined from the global auroral observation and identified as the initial brightening of an auroral arc; the local CD onset is marked by the start of a strong and abrupt magnetic dipolarization, typically with $>10 \mathrm{nT}$ change in $B_{x}$ (when the probe is off the neutral sheet) within tens of seconds, accompanied with a substantial drop of total pressure.

\subsection{March 2007 event}

Donovan et al. (2008) reported a small substorm event on 13 March 2007. This auroral breakup has a relatively smooth start of intensification from 05:07 to 05:08 UT while the best estimation of the onset time is about 05:07:45 UT. The initial brightening occurred on the part of the growth phase arc closest to a proton aurora arc. Azimuthal wave-like intensification structure (longitudinal modenumber $\sim 120$ ) arose along the arc during the EP. THEMIS satellites were in the coast phase during this event in a pearls-on-string formation (see Fig. 1 in Donovan et al., 2008, for probe location). The ESA was only operational at TH-A, the outermost of the three clustered probes $\mathrm{D} / \mathrm{B} / \mathrm{A}(L \sim 8.5)$, with unreliable ion flow data. The top three panels of Fig. 1 give the FGM measurements from TH-D/B/A probes. The magnetic fields are plotted in VDH coordinates, where the $\mathrm{H}$ axis is antiparallel to the Earth's dipole axis, the V-axis is radially outwards and is parallel to the magnetic equator, and the $\mathrm{D}$-axis completes the right-hand orthogonal system. The CD is clearly manifested by a precipitous drop of $B_{v}$ component on all satellites in a sequence D->B->A. An abrupt decrease of total pressure (bottom panel) was also seen on TH-A concurrently with the $B_{v}$ drop at 05:08:45 UT, confirming a substantial 


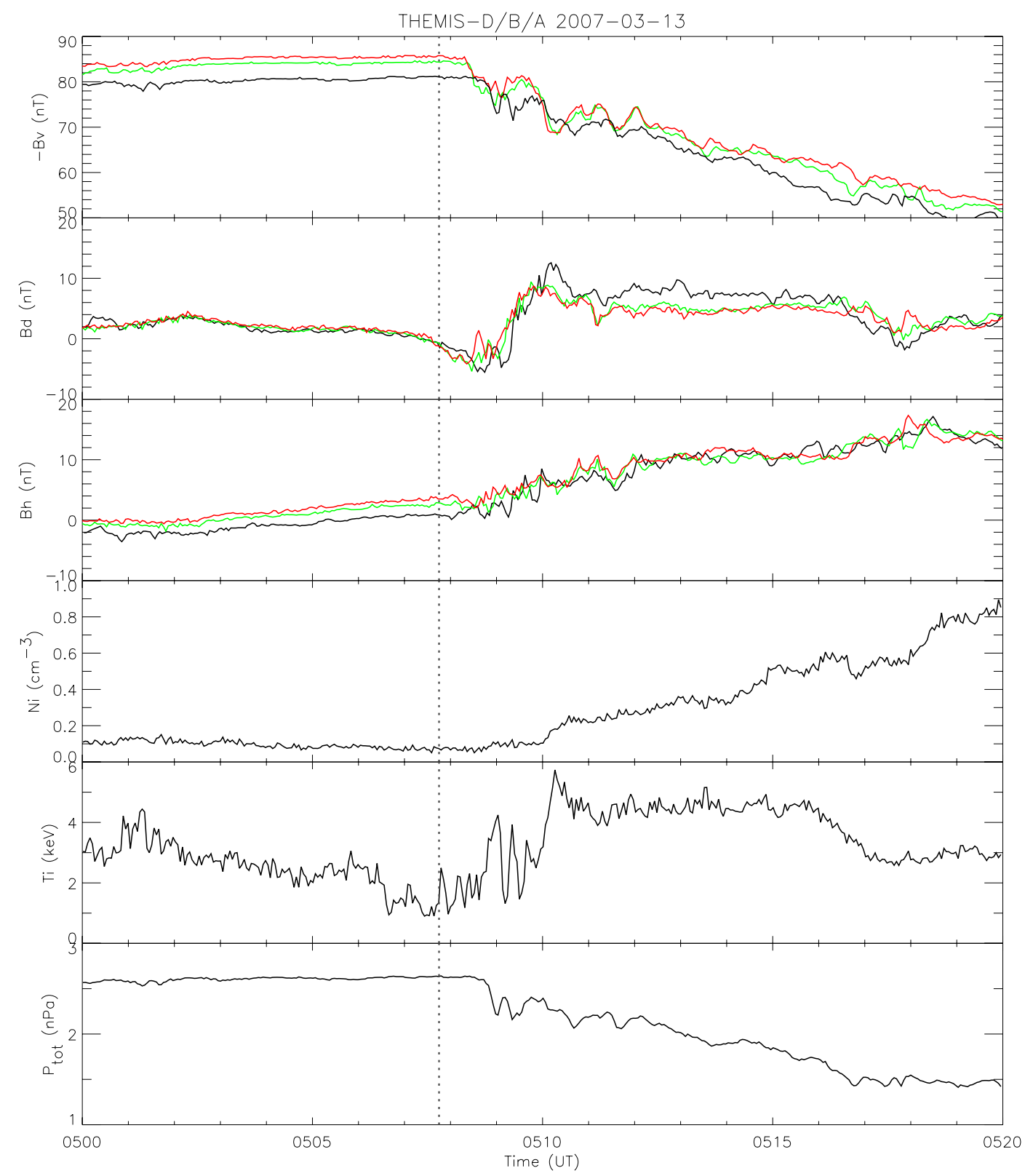

Fig. 1. THEMIS observations for 13 March 2007 event. The first three panels give the magnetic fields in VDH coordinates from TH-D (red), TH-B (green), and TH-A (black) probes. The 4th to 6th panels show the ion number density, ion temperature and total pressure on TH-A, respectively.

reduction of cross-tail current intensity. This sequence was examined by Donovan et al. (2008) in conjunction with the ground ASIs and found to indicate a dominantly radial propagation of CD fronts with an outward speed of $\sim 100 \mathrm{~km} / \mathrm{s}$. Panels 4 and 5 show the ion number density and temperature. It is interesting to note that, of all the macroscopic plasma parameters the ion temperature exhibits the most dynamic variations in 1-2 min prior to the EP onset and local CD. It dropped from $\sim 3 \mathrm{keV}$ to slightly below $1 \mathrm{keV}$ after 05:06 UT, and rose back to $\sim 5 \mathrm{keV}$ after the local $\mathrm{CD}$, in an oscillatory pattern.
Since the main interest of this paper is the ULF wave activity in the Pi1 and Pi2 bands, we apply a Savitzky-Golay filter (Savitzky and Golay, 1964) to separate out the "ambient" field variation before the wavelet decomposition. Since this procedure is applied throughout this study, we illustrate its use in Fig. 2. The observed magnetic field components in shown in black lines, and the "ambient" field variations obtained from 60-point (in 3-s data interval) SavitzkyGolay smoothing are shown in green lines; their difference is subject to a Morlet wavelet analysis for time-frequency decomposition shown in the scalograms. The procedure is 


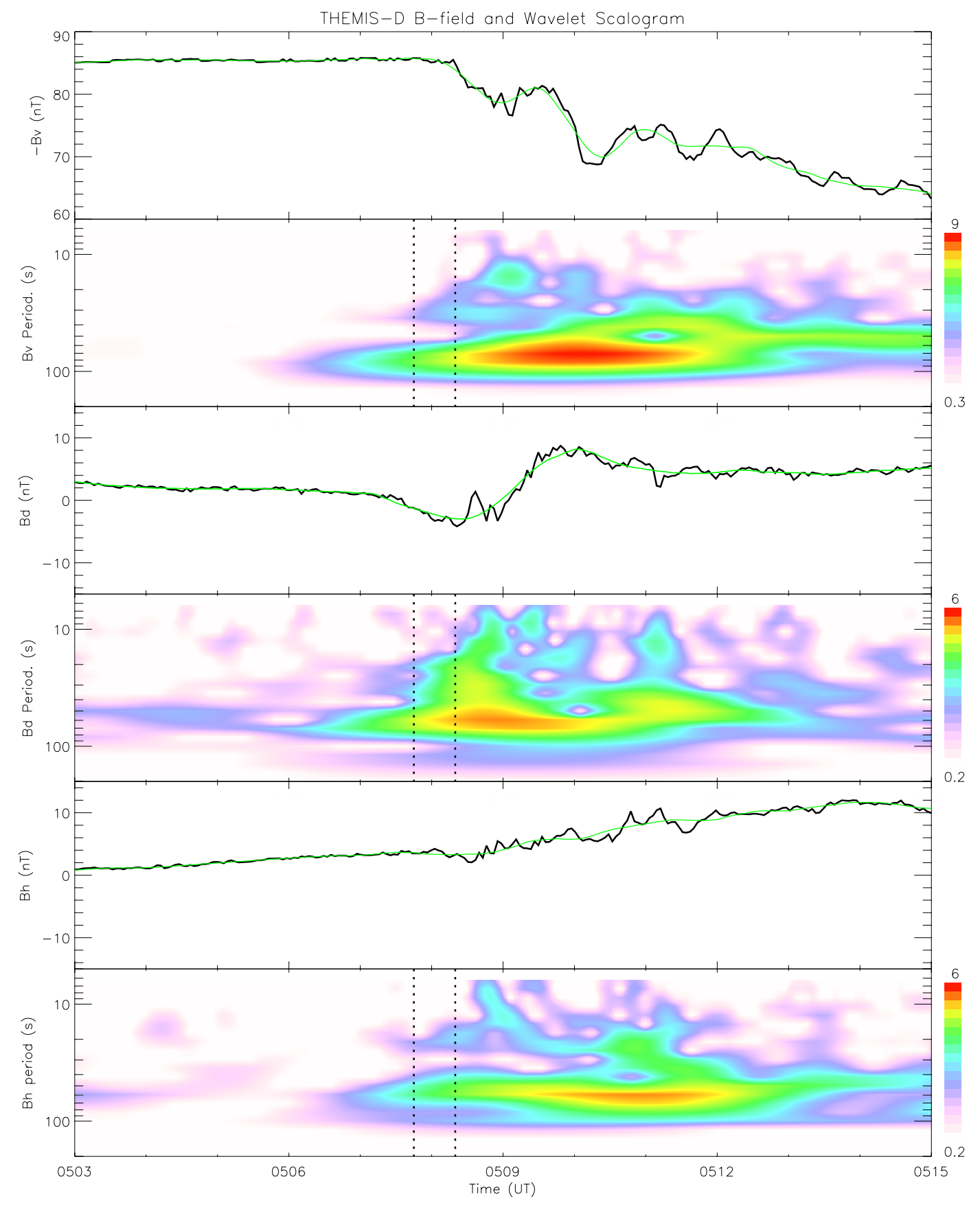

Fig. 2. The figure is grouped into $B_{v}, B_{d}$ and $B_{h}$ components observed on TH-D. In each group the upper plot shows the observed magnetic field component (black), the "ambient" field variation (green) obtained from Savitzky-Golay smoothing. The bottom panel shows the wavelet scalogram of the magnetic field components. Two vertical lines indicate the EP onset time and the local CD onset time, respectively.

equivalent to a high-pass detrending filter but in our experience works better in the presence of large-amplitude stepwise "jump" (i.e., the dipolarization) in the raw data. While there is no straightforward way to entirely separate the alias of those transient "jumps" from the actual wave components, the guideline applied throughout the study is: when in doubt, check with the original data whether the "waves" are visually noticeable.
Figure 2 shows that after $\sim 05: 06 \mathrm{UT}$, ULF wave activity first enhanced at period range $60-100 \mathrm{~s}$. Such gradual intensification occurred around 1 min before the substorm EP onset (first vertical line). Those Pi2 waves formed a continuous band lasting for many minutes, with strong intensification after the local CD onset. Higher frequency components in Pi1 band emerged in conjunction with the substorm EP onset. Looking back into the original data we confirm those waves 


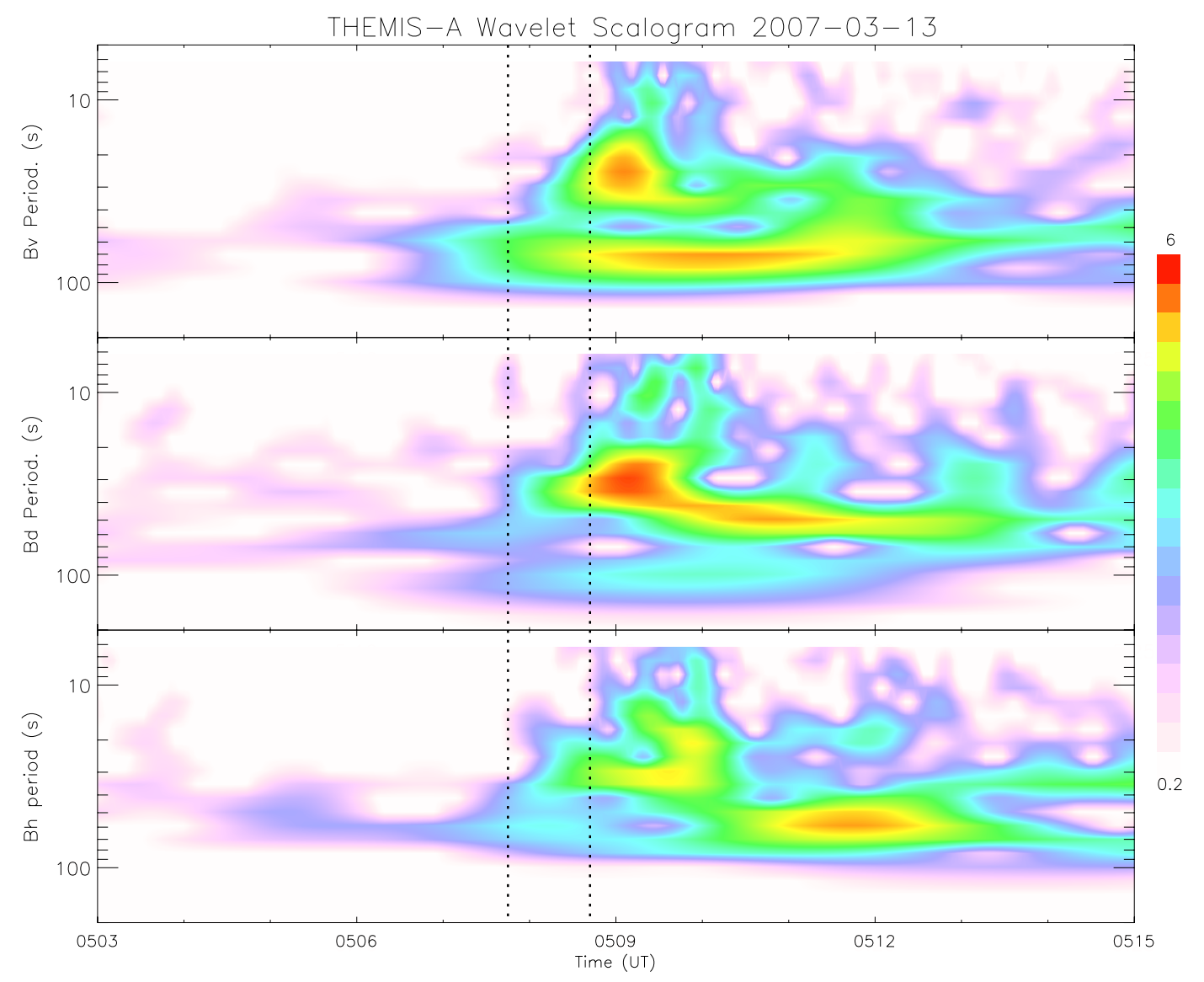

Fig. 3. Wavelet scalogram of the magnetic fields observed on TH-A. Two vertical lines indicate the time of EP onset and local CD onset time, respectively.

are realistic since the small-amplitude oscillations at 10-20 s periods are obviously embedded in all magnetic field components. Seen from the scalogram of $B_{d}$ (azimuthal) component, the Pi1 waves appear in a stepwise manner. Roughly concurrent with the substorm EP onset, the upper frequency band abruptly extended to $\sim 0.05 \mathrm{~Hz}(20 \mathrm{~s})$; while shortly after the local $\mathrm{CD}$ onset the wave bands further extended to $>0.1 \mathrm{~Hz}$. Those higher-frequency Pi1 waves, however, is intermittent and shorter-lived compared to their Pi2 counterpart.

Figure 3 shows the scalogram of TH-A magnetic field. The overall pattern resembles that from TH-D, i.e., the leading $\mathrm{Pi} 2$ band waves that appeared before to the onset and lasted over the entire EP; the emergence of a higher frequency mode immediately after the EP onset and local CD. The only difference is that the $\mathrm{CD}$ onset lags that of $\mathrm{TH}$ D by $\sim 24 \mathrm{~s}$ and thus the appearance of $>0.1 \mathrm{~Hz}$ waves occurred later accordingly. Another noteworthy feature is that the emergence of the $\sim 60$ s period waves is roughly concurrent with the ion temperature drop from ESA survey mentioned above.

\subsection{February 2008 event}

The second substorm event of interest occurred around $\sim 07: 00$ UT on 13 February 2008. During the event interval TH-A and $\mathrm{C}$ are on the dusk flankside magnetosphere, and TH-B reside in the mid-tail $(L \sim 30)$ lobe. We shall focus on two probes in the near-Earth plasma sheet, TH-D and $\mathrm{E}$, which were close to each other in terms of radial distance while separated by $\sim 0.9 R_{E}$ in azimuth (see the top of Fig. 6 for exact coordinates). The satellite geometry thus implies that the time delay between TH-D and E we shall repeatedly see in a few pieces of observations hints an azimuthal (westward) expansion of the onset-related instability.

Figure 4 gives an overview of the event from auroral and magnetic observations. The top panel is the keogram of the THEMIS ASI, while the other three show the magnetometer observations from Fort Churchill, Gillam and Rabbit Lake stations. This substorm event is not intense in terms of ground magnetic disturbances $(\sim 100 \mathrm{nT})$ but with very welldefined auroral breakup feature, with estimated onset time at 06:57:45 $\pm 3 \mathrm{~s}$ from THEMIS ASI observations. 

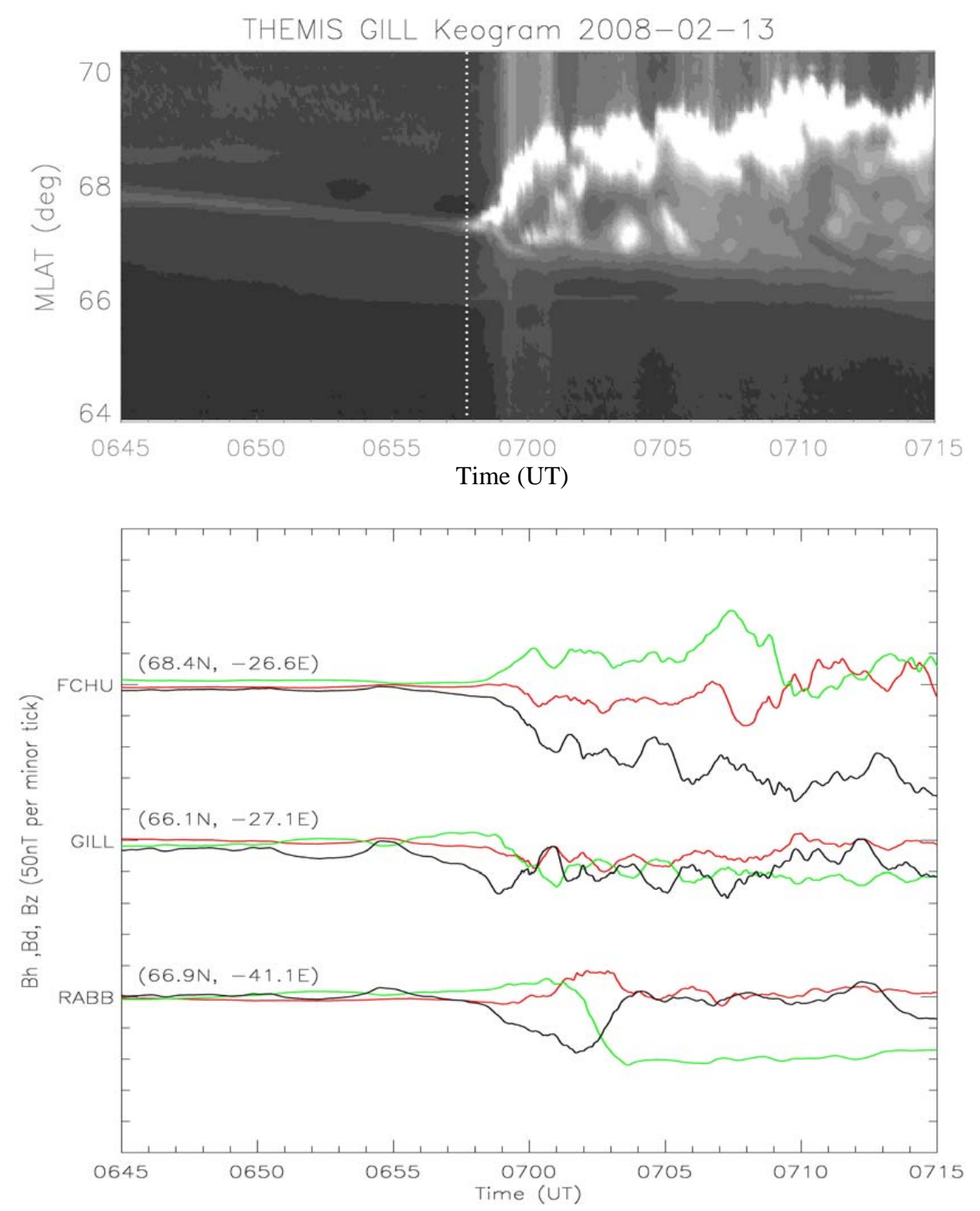

Fig. 4. The first panel show the keogram of THEMIS GILL ASI; The rest panels shows the magnetograms from FCHU, GILL and RABB stations. Black, green and red lines denote the $\mathrm{H}, \mathrm{D}$, and $\mathrm{Z}$ components of the ground magnetic field.

Figure 5 gives a series of selected images revealing the initial brightening and subsequent expansion of the substorm aurora obtained from THEMIS ASI at Gillam. The ionospheric footprint of TH-D and E estimated from Tsyganenko89 model are also plotted for reference. The activation began on a preexisting arc at $66.6^{\circ}$ MLAT which had been slowly intensifying within a couple of minutes before the onset, reflected in the ground magnetogram as a slow decrease of $\mathrm{H}$ component since 06:55 UT. The major brightening began after 06:57:45 UT. In the typical of EP onset as illustrated in Liang et al. (2008), the intensification quickly evolved into a number of azimuthally-spaced spots longitudinally centered about TH-D, which became quite visible at 06:57:54 UT. In the following frames we provide the evolution of those lon- gitudinally wave-like aurora structures and their prominent westward expansion. New structure appeared successively west to the previous one, hinting at a propagating wave mode associated with the substorm expansion. Little eastward expansion was found. The azimuthal "wavelength" estimated from the longitudinal separation of the intensified structures is about $1.6-2.4^{\circ} \mathrm{MLON}$, or $70-105 \mathrm{~km}$ in terms of the spatial distance. After 06:08:15 UT poleward auroral expansion as well as moderate eastward expansion began to develop. The poleward expansion was far from uniform along the initial breakup arc, instead, a segment longitudinally close to TH-D was first significantly elevated in latitude while the rest only slightly broadened by 06:58:33 UT. Embedded azimuthal wave-like structures were still quite visible but with 


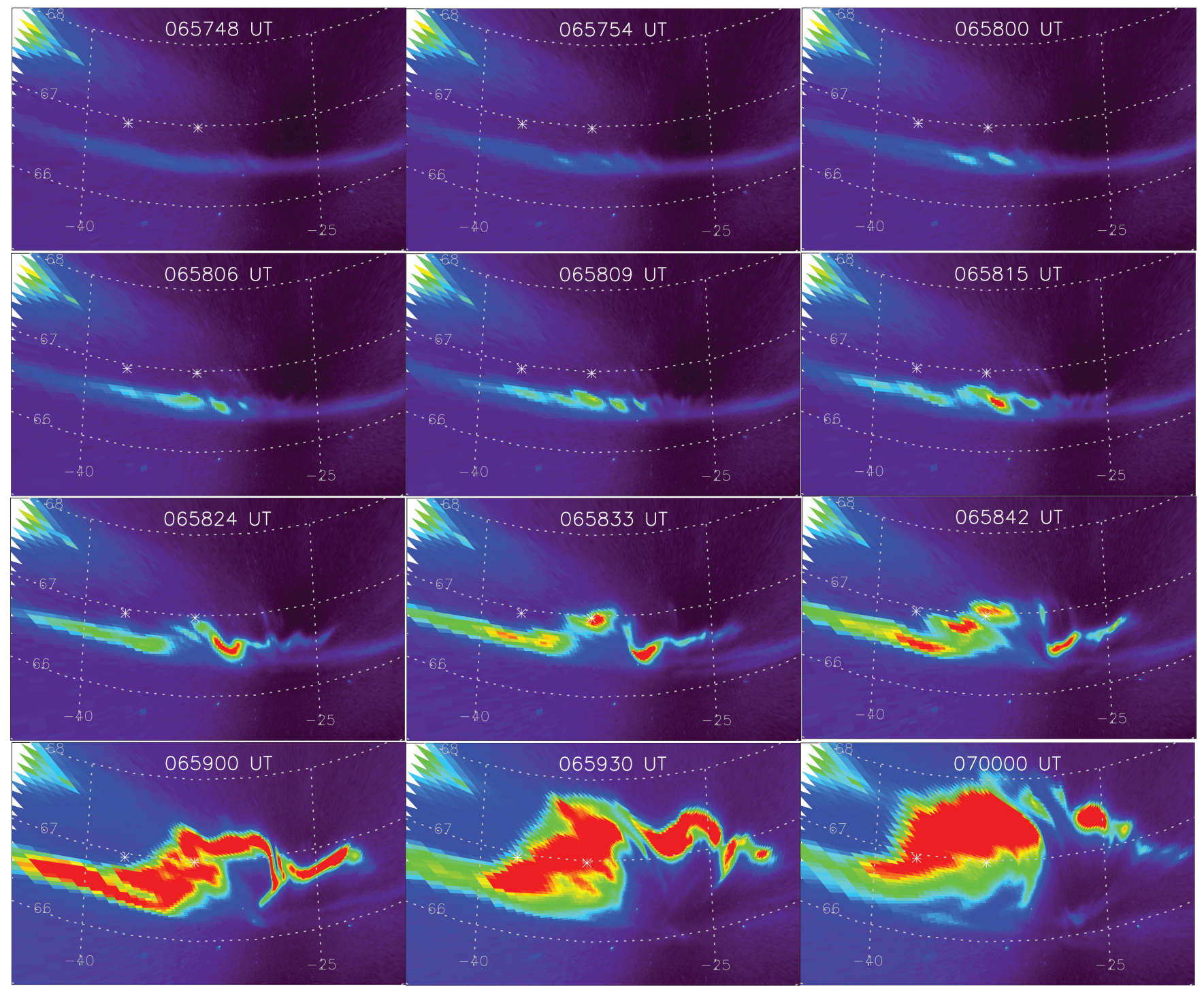

Fig. 5. A series of auroral images showing the auroral breakup and expansion. The footprints of TH-D (eastern one) and TH-E are labeled in asterisks.

somewhat larger wavelength as compared to, e.g., the previous 06:58:09 frame. The poleward expansion gradually evolved westward toward TH-E; a distinct aurora bulge was formed by 06:59 UT. Active intensification and overall expansion continue to proceed in the following minute and the auroral bulge expanded beyond $68^{\circ}$ MLAT at 07:00 UT.

Figure 6 gives the in-situ observation from TH-D and E probes. In this event the full set of THEMIS instruments were operational, including FGM, ESA, and EFI. The first three panels give the magnetic fields. All satellites are south of the neutral sheet (since $B_{x}<0$ ). The local CD was observed on TH-D/E by a strong decrease of $\left|B_{x}\right|$ as well as the total pressure (the 9th panel) at $\sim 07: 00 \mathrm{UT}$, about $2.2 \mathrm{~min}$ after the auroral onset. If we apply the outward CD propagation speed of $\sim 100 \mathrm{~km} / \mathrm{s}$ estimated from the pervious event, and assume an Alfvénic travel time (i.e, the delay between the disruption in the magnetosphere and the auroral breakup in the ionosphere) of $\sim 1 \mathrm{~min}$, the initial onset would be reasonably placed at $L \sim 8 R_{E}$. Although there is no distinct difference in terms of the start time of CD between TH-D and TH-E, the rise of $B_{x}$ is stronger and more abrupt on TH-D, while milder and more gradual on TH-E, implying that THD was presumably located closer to the center of the current disruption region which can be partly inferred from the above auroral observations. The following three panels give the ion flow velocity. We see no hint of either earthward or tailward fast flows prior to the onset. Together with the ground ASI observations that there was no discernible poleward auroral activation and/or streamer structure minutes prior to the breakup we conclude that this substorm was not likely a consequence of reconnection-driven fast flows. 


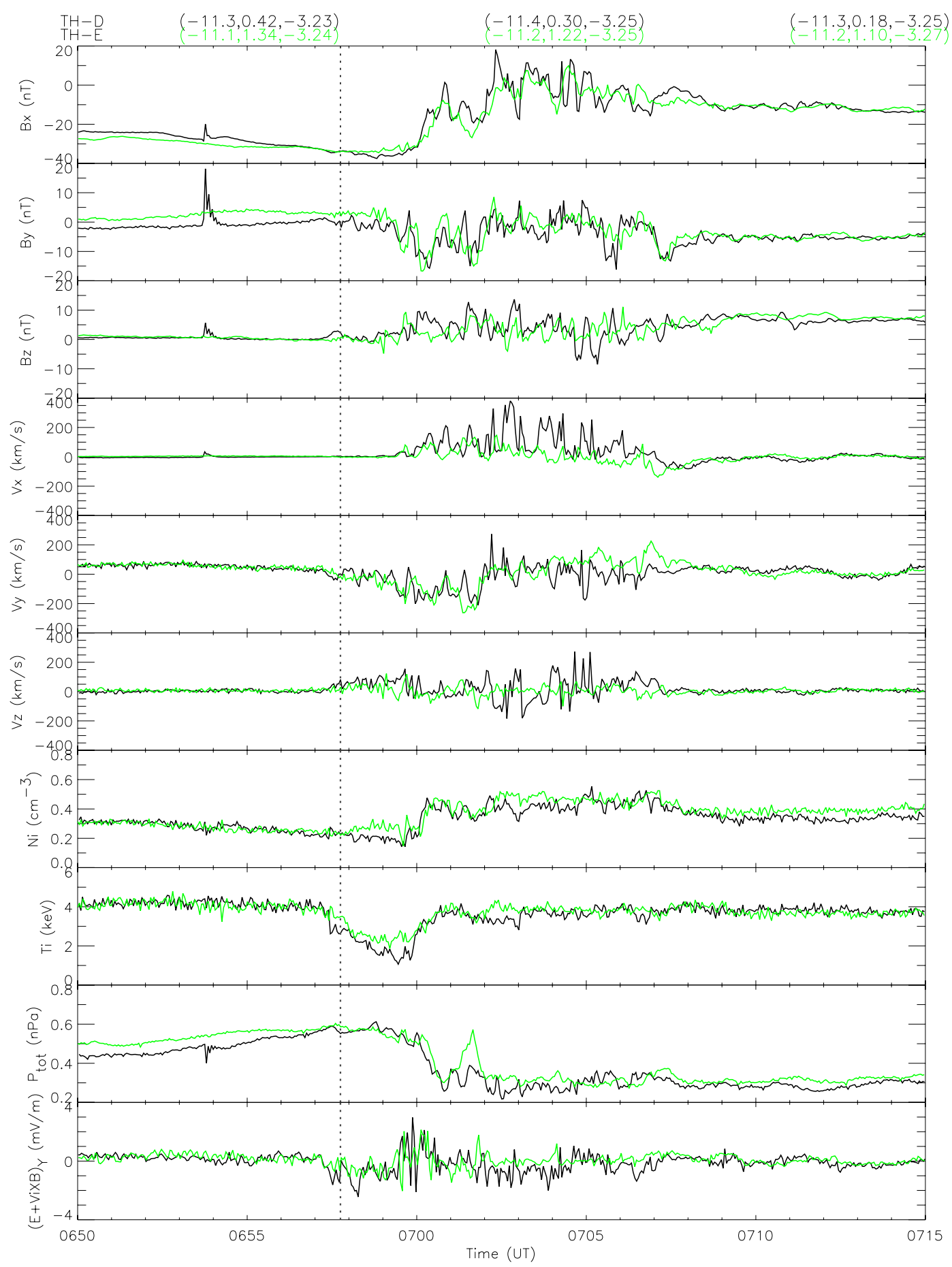

Fig. 6. THEMIS observations for 13 February 2008 event. From top to bottom are the three magnetic field components in GSM coordinate, ion flow velocities in GSM coordinate, ion number, ion temperature, total pressure and the DSL-Y component of $\boldsymbol{E}+\boldsymbol{u}_{i} \times \boldsymbol{B}$ from TH-D (black) and TH-E (green) FGM, ESA \& EFI observations.

Panels 7 and 8 give the ion density and thermal temperature. We again see that in a couple of minutes prior to the local $\mathrm{CD}$ onset the most noticeable variation of plasma parameters comes from the ion temperature. Starting from $\sim 06: 57 \mathrm{UT}$ the ion temperature dropped from 4 to $1 \mathrm{keV}$ as seen on THEMIS D, and restored to $\sim 4 \mathrm{keV}$ immediately following the current disruption at $\sim 07: 00$ UT. The ion temperature drop was also seen on TH-E but was less dramatic in 

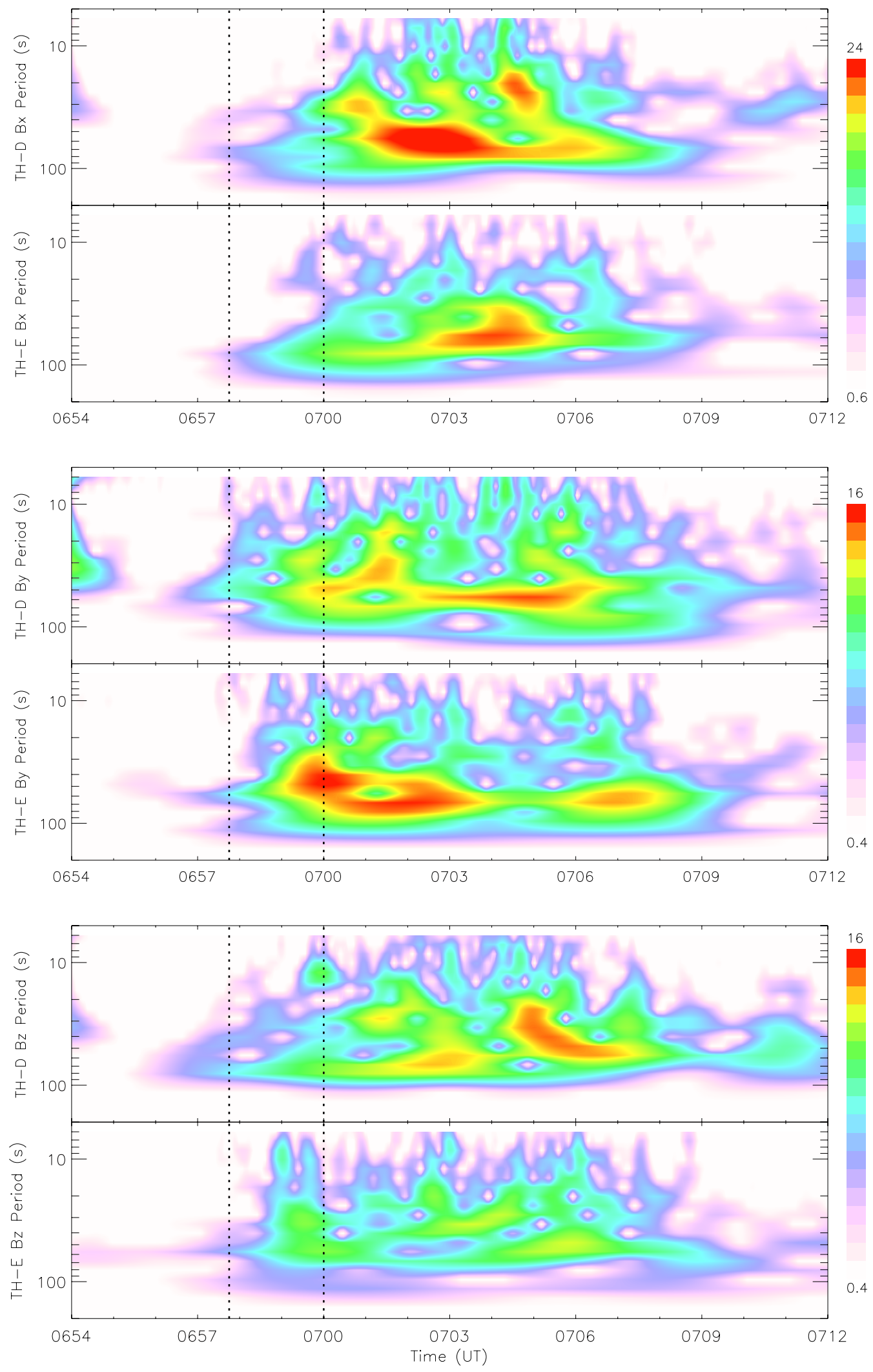

Fig. 7. Wavelet scalogram of the magnetic fields observed on TH-D and E. Two vertical lines indicate the time of EP onset and local CD onset, respectively. 


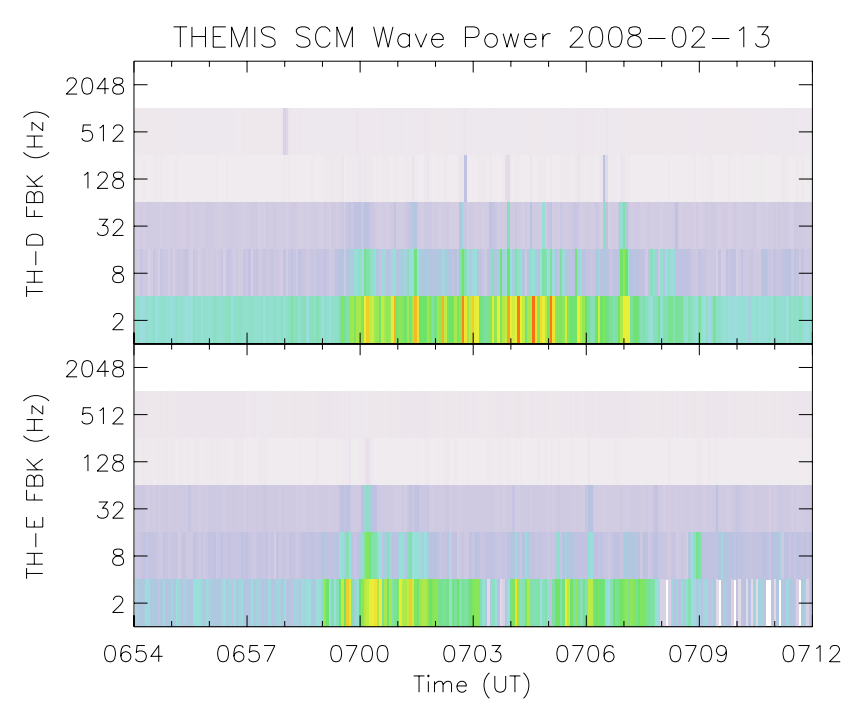

Fig. 8. THEMIS SCM measurements of the high-frequency wave power on TH-D (upper panel) and TH-E (bottom panel).

the drop level and lagged that on TH-D, again hinting a westward propagation effect. The bottom panel shows the electric field in the plasma frame of reference, $\boldsymbol{E}+\boldsymbol{u}_{i} \times \boldsymbol{B}$, which would be zero in the ideal MHD approximation. Only the Ycomponent in a despun satellite (DSL) coordinate is shown, which is approximately along the GSM-Y direction during the event interval. It is interesting to note that, concurrent with the ion temperature drop there is a substantial wave-like deviation from the frozen-in-flux condition. The observation indicates the presence of non-MHD process minutes prior to the local CD.

Figure 7 gives the wavelet scalogram of the FGM data for TH-D/E. The results show an overall similarity to Figs. 2 and 3. Around 06:57 UT1 Pi2 band at period 40-60 s emerges mainly on the $B_{y}$ (and weaker on $B_{z}$ ) component, hinting that the wave is likely Alfvénic in nature. We notice again that the initial enhancement of the Pi2 wave activity was close in timing to the commencement of the ion temperature drop effect but preceded the EP onset and local CD. Higher frequency component at $\sim 20$ s period quickly emerged subsequently, almost concurrent with the EP onset on TH-D and a few tens of seconds later on TH-E as seen on the $B_{y}$ component. We look back to Fig. 6 and confirm conspicuous wave oscillations appearing on $B_{y}$ component at the EP onset. Even higher frequency component at period $\leq 10 \mathrm{~s}$ emerged in conjunction with the local CD. Similar to the previous event we again notice that the waves at $\mathrm{Pi} 2$ range were more continuous and long-standing, while the waves at Pi1 range were more intermittent and shorter-lived.

The FGM data used above are limited by the spinresolution and thus the wave analysis is subject to a Nyquist frequency $1 / 6 \mathrm{~Hz}$. Since the CCIs (Lui et al., 1991), another main branch of onset instability candidate, are featured with frequency above the ion gyrofrequency $(\sim 0.5 \mathrm{~Hz}$ prior to the local CD and $\sim 0.14 \mathrm{~Hz}$ shortly afterwards for this event), it would be of particular interest to investigate the waves at such high frequency. The THEMIS searching coil magnetometer (Roux et al., 2008) is capable of providing magnetic field fluctuations/waves up to $4 \mathrm{kHz}$. The THEMIS FBK dataset contains the post-processed wave power at 2, 8, 32, 128,512 , and $2048 \mathrm{~Hz}$ and is presented in Fig. 8. We see that intensification of waves with frequency $>1 \mathrm{~Hz}$, together with the upper edge of Pil band (6-10s) shown in Fig. 7, started only in the very vicinity of the local CD onset (within tens of seconds), and disappeared after the large-amplitude dipolarization was over after $\sim 07: 06$ UT. The observations indicate that high-frequency instability such as the CCIs might be a viable candidate for the $\mathrm{CD}$ excitation, but is only a local and immediate process with little spatial propagation; otherwise one would expect to observe the waves at times other than the local CD, as a remotely propagated effect from other CD sources, which will be discussed in the next section.

\section{Discussion}

Through two substorm events with coordinated groundbased and in-situ observations, we have identified a few key features of ULF wave activities a few minutes before and after the substorm EP onset and local CD. We revealed that a Pi2 band wave (40-100 s) usually appears 1-2 min before the substorm EP onset, while higher-frequency wave, nominally at Pi1 band (10-30 s), emerge within few tens of seconds after the EP onset and intensifies during the local CD. Those features are repeatable in many of substorm events we surveyed (e.g., Liu et al., 2008). The readers are also referred to Lui and Najmi (1997) and all events presented by Saito et al. (2008a, b) to see similar features, e.g., the leading Pi2 waves and intermittent Pil waves accompanying the local $\mathrm{CD}$. This paper, in conjunction with a number of other related studies by Liu et al. (2008); Liu and Liang (2009), and Liang et al. (2008), is part of our efforts to thread all the key wave characteristics, in-situ and ground observed, toward a synthesis of plasma wave modes responsible for the substorm EP onset and local CD.

The Pil band waves arising with the substorm EP onset and local CD coexist with the Pi2s but exhibit totally different temporal behavior. Examining the spectrum we can first eliminate the possibility that the Pi1 is merely a high-order harmonics of the Pi2, for otherwise they must have similar temporal pattern which is patently not so. We then eliminate the possibility that the observed Pil wave is merely a Doppler-shift of the preexisting Pi2 band; for otherwise the Pi1 would replace the Pi2 as the main band. All evidences uncontrovertibly suggest that the Pi1 and Pi2 bands are characteristic of fundamentally different plasma wave modes initiating at different stages of a substorm. 
The wave activity of our interest starts with the appearance of Pi2 band 1-2 min before the substorm EP onset. In event 1 with multi-satellite geometry an "inside-out" sequence of substorm expansion and waves was unambiguously identified (Donovan et al., 2008). In event 2 we do not have any evidence of fast flows prior to the onset either. This leads us to believe that the waves stem from internal instability. The emergence of Pi2 band waves a few minutes before the substorm dipolarization onset was at times revealed from the GOES measurements (Holter et al., 1995; Arnoldy et al., 1998). Pi2 waves at $\sim 60 \mathrm{~s}$ period $1-3 \mathrm{~min}$ prior to the substorm EP onset were also identified from GEOTAIL measurements by Saito et al. (2008b), and proposed as the manifestation of the ballooning mode. In the following discussion we shall follow this notion, although we admit that there are other Pi2 generation sources, particularly after the EP onset when the Pi2 band noticeably broadened, such as a standing Alfvénic mode trapped within current sheet (Holter et al., 1995). Our observations are consistent with Saito et al. (2008b) except that in their events $\sim 60$ s period pre-onset waves are most prominent on $B_{x}$ component since their event selection criteria ensured that the satellite (GEOTAIL) is located very close to the neutral sheet, while in many THEMIS events the satellite is fairly off the neutral sheet such that the pre-onset $B_{y}$ waves become more pronounced, consistent with what would occur as a result of the Alfvénic nature of the ballooning mode. The ballooning is in principle a coupling between the slow mode (which releases the free energy embedded in the plasma pressure distribution) and the shear Alfvén mode (which transfers the energy to the ionosphere). In terms of the magnetic effect, the ballooning mode near the equatorial high- $\beta$ plasma sheet acts to modulate the magnetic field curvature and thus affect mostly the $B_{x}$ component, while remote effects via Alfvén waves and the accompanying FACs dominate away from the neutral sheet. Therefore one should expect that for satellite off the neutral sheet the $B_{y}$ component from in-situ observation best manifested the Alfvénic nature of the ballooning mode and its interaction with the local current sheet. Furthermore, owing to its Alfvénic nature the ballooning mode may drive FACs (Pu et al., 1997) that possibly related to the very initial brightening and structuring of the breakup arc.

As a low-frequency mode the ballooning "wave" observed a satellite is typically a Doppler-shifted, with $\omega \approx k_{y} \cdot u_{y}$ in the satellite frame of reference, where $k_{y}$ and $u_{y}$ denote the azimuthal wavevector and the ambient azimuthal flow, respectively. Under this notation Saito et al. (2008b) inferred the azimuthal wavelength of the ballooning mode to be 600$3000 \mathrm{~km}$, comparable to the ambient ion gyroradius. Interestingly, Liang et al. (2008) using THEMIS ASI observations independently derived roughly the same wavelength estimate. We shall test this correspondence in event 2 with ion flow observations. On the first emergence of the ballooning mode before 06:57 UT its frequency is centered at 5060 as seen from Fig. 7; by that time the ambient aziumthal flow $u_{y}$ on both TH-D and $\mathrm{E}$ is stable at $40 \pm 5 \mathrm{~km} / \mathrm{s}$ from ESA measurements. We thus obtain an estimation of 1750$2700 \mathrm{~km}$ as azimuthal wavelength. We estimate the longitudinal mapping factor as $1^{\circ} \approx 1100 \mathrm{~km}$ based upon the T-89 model according to the actual satellite positions. The above values well match the longitudinal wavelength $1.6-2.4^{\circ}$ inferred from ground ASI observations at around 06:58 UT in event 2, with a nominal Alfvénic transit lag of $\sim 1 \mathrm{~min}$. The consistency suggests that the longitudinal wave-like structures during the initial auroral brightening as a common feature reported by Liang et al. (2008) are possibly manifestations of the growth of a ballooning mode. A more general survey of similar events with conjugate ground-based and insitu THEMIS observations is undergoing and will be the contents of future publication. We realize though, there are other candidate mechanisms to account for the quasi-periodic auroral structures. For example, the large-sale auroral spirals $(\lambda \sim 100-400 \mathrm{~km}$, see Murphree et al., 1994) and small-scale auroral curls $(\lambda \sim 1-8 \mathrm{~km}$, see Trondsen and Cogger, 1998) were interpreted as the manifestation of a shear flow-related Kelvin-Helmholtz instability. We particularly note that the wavelength range identified by Murphree et al. (1994) is on average larger than, but certainly might overlap with, that from our THEMIS onset observations $(\lambda \sim 40-130 \mathrm{~km}$, see Liang et al., 2008). We acknowledge that so far our identification of the ballooning mode is somewhat tentative, and we do not deny the possibility of the K-H instability being a candidate mechanism for the observed auroral structures. We hereby point to one other possibility that the K-H instability may coexist and couple with the ballooning to become a "shear flow ballooning mode" (Voronkov et al., 1997, 2000).

The ballooning instability has been considered by a number of researchers as the underlying mechanism of rapid thinning of the current sheet within the final minutes prior to its disruption (Liu, 1997; Cheng, 2004). Physical detail of such interaction is not entirely clear so far. We showed in two events that the ion temperature underwent a precipitous drop from $3-4 \mathrm{keV}$ to $\sim 1 \mathrm{keV}$ following the appearance of the ballooning mode. From our survey of THEMIS events we find that the ion temperature drop (ITD) minutes prior to the local CD onset is a common feature when the probe is at the current sheet boundary. This feature is interpreted as an indication of a dramatic current sheet thinning such that the probe underwent a transition from the plasma sheet into the boundary layer and/or the lobe. When the thinning proceeds to the order of thermal ion gyroradius kinetic effects arise. The deviation from the frozen-in flux condition revealed by the bottom panel of Fig. 6 again gives clues that the current sheet has been thinned to an ion kinetic scale. We also note that in event 2 a few negative wiggles on the Y-component ion flow $u_{y}$ appeared after the arrival of the ballooning mode ( $\sim 06: 57$ UT) and maximized in magnitude at the local CD. Those negative excursions of $u_{y}$ are consistent with the incidence of a wave train of quasi-electrostatic electric field pointing towards the neutral sheet, forming out of a charge 
separation due to the ion demagnetization in an extremely thinned current sheet (e.g., Asano et al., 2003). The quasielectrostatic wave is also frequently observed minutes before the local CD onset, and is proposed as a manifestation of the interaction between the kinetic ballooning mode and the local current sheet (Liu et al., 2008). A statistical study on the ITD effect, the quasi-electrostatic electric field, and their possible relationship to the ballooning mode from 20 substorm events are the contents of separate paper (Liang et al., 2009). Eventually, sudden collapse of current/magnetic field occurs along a segment of the TCS which becomes unstable and is subject to abrupt disruption, as a result of above interaction process.

Recently, Liu and Liang (2009) applied the Hall-MHD equation set to the perturbation of a one-dimensional equilibrium thin current sheet (TCS), and investigated analytically and numerically the eigenvalue problem, involving the frequency, the growth rate, and the azimuthal wavevector $\left(k_{y}\right)$ of the instability mode of a TCS. They found that the instability threshold is highly contingent upon of the profile (more exactly, the second-order derivative) of the ion azimuthal drift across the thickness of the TCS; the presence of a quasielectrostatic E-field and/or a drop of ion temperature at the current sheet boundary both make the TCS more prone to instabilities. This two-fluid instability mode of Liu and Liang (2009) is characterized with period range 10-30 s, well consistent with the observations presented above. We shall term it as TCS mode hereafter. Such Pi1 mode is propagational; in satellite observations it reveals themselves first as a remotely propagated effect presumably from the onset origin, and then occurring in-situ when the local CD is excited.

The tailward propagating feature of the TCS mode is not handled in the current theory in Liu and Liang (2009) due to the neglect of $k_{x}$ (i.e., tailwise wavevector) for the sake of mathematical simplicity. We however, speculate the possibility that when a TCS mode propagating outward from its initial excitation region (i.e., the onset place) it may degenerate via mode conversion to other wave modes, which is essential for these Pi1 waves to be remotely detected either earthward or tailward of the onset origin. For an earthward propagation one may expect that the Pil waves are coupled into a fast compressional mode, which may account for the observations of Pi1 waves on GOES satellites at geosynchronous distance (Arnoldy et al., 1998). In an event study Lessard et al. (2006) provided evidences that the GOES Pi1 waves were compressional in nature. Similarly, it is reasonable to expect that those Pi1 waves may also couple to rarefaction waves during its downtail passage. It has long been speculated that the initial disturbance at the onset location emanates a tailward-propagating rarefaction wave and produces a chain effect to make the downtail current sheet become more unstable (Lui, 1991). However, while those waves may act as a catalyst the successive evolution of the local current sheet and magnetic field structure towards eventual destabilization, which determines the propagation of the $\mathrm{CD}$ front, is in gen- eral much slower. In both events reported (and also in many similar events form our survey) the Pi1 waves appear within tens of seconds after the substorm EP onset, indicating that the propagation delay from the onset location to the satellite roughly matches the Alfvénic travel time to the auroral ionosphere. The local CD, however, is often observed as lagging the substorm EP onset by minutes (e.g., Liu et al., 2008). The observations suggest that the tailward propagation of $\mathrm{CD}$ and that of the Pi1 waves must travel at different speeds. Assuming the wave and the $\mathrm{CD}$ are of the same origin (i.e., the onset place) and both propagate tailward, we have the following mathematical relationship,

$\left(T_{\mathrm{Pi} 1}-T_{\mathrm{EPO}}+T_{\mathrm{ALf}}\right) * V_{\mathrm{Pi} 1}=\left(T_{\mathrm{CD}}-T_{\mathrm{EPO}}+T_{\mathrm{ALf}}\right) * V_{\mathrm{CD}}$

where $T_{\mathrm{Pi} 1}, T_{\mathrm{CD}}$ denotes the arrival time of the Pil waves and the $\mathrm{CD}$ from in-situ observation,, respectively, $T_{\mathrm{EPO}}$ is the EP onset time determined form the auroral observation, and $T_{\mathrm{Alf}}$ is the Alfvénic travel delay. $V_{P i 1}$ and $V_{c d}$ denote the propagation speed of waves and the $\mathrm{CD}$ front, respectively. We use TH-D observation in event 2 for sample calculation. Seen from Fig. 5 that TH-D was located longitudinally within the initial onset region as well as center of the poleward-expanding auroral bulge (disruption region) thus little azimuthal propagation delay is expected (see discussion below). The local CD occurred at 07:00 UT while the EP onset was at about 06:57:45 UT, seen from the $B_{y}$ component scalogram the $15-25 \mathrm{~s}$ period waves appeared within $\sim 10 \mathrm{~s}$ after the onset. Assuming a typical vale of $T_{\mathrm{Alf}}=1 \mathrm{~min}$ in the near-Earth tail, we obtain from Eq. (1) $V_{\mathrm{Pi} 1} \approx 3 V_{\mathrm{CD}}$. The wave propagation is thus much faster than the $\mathrm{CD}$ front propagation. Using the value $V_{\mathrm{CD}} \sim 100 \mathrm{~km} / \mathrm{s}$ inferred from event 1, we may reasonably place the onset origin at $L \sim 8 R_{E}$ and obtain a wave propagation speed of $\sim 300 \mathrm{~km} / \mathrm{s}$, comparable to other estimated rarefaction wave speed from theories and observations (Ohtani, 1993; Saito et al., 2008a). The variability of Alfvénic travel time should not drastically alter the above estimation. An extension of the theoretical work of Liu and Liang (2009) to take into account finite $k_{x}$ will be carried out in the near future.

For the two events presented the probes under investigation were longitudinally close to the onset meridian as inferred from the global auroral images. The scenario undoubtedly becomes more complicated for probes longitudinally away from the onset sector such that the azimuthal propagation effect may also play a role. In event 2 we have noted that common wave bands around $\sim 20 \mathrm{~s}$ period were seen on both THEMIS probes, almost concurrently with the EP onset on TH-D but obviously lagged on TH-E. For a more quantitative estimation we cross-correlate the wave power between $0.05 \pm 0.01 \mathrm{~Hz}$ on two probes during 06:57-07:00 UT, and obtain a delay of $\sim 33 \mathrm{~s}$ from TH-D to E. TH-D and E are separated mainly in Y-direction by $\sim 0.9 R_{E}$; the small yet non-negligible tailwise separation $\left(\sim 0.2 R_{E}\right)$ between the probes would contribute $\sim 4$ additional seconds using the above-estimated tailward propagation speed. We thus infer 
a duskward propagation speed of $\sim 150 \mathrm{~km} / \mathrm{s}$. Caution must be taken in that the timing on the $B_{y}$-wave component cannot be used to estimate the azimuthal prorogation speed of the wave itself, but more likely represent a spread speed of the source of the wave. Coincidentally, Liu and Liang (2009) predicted an azimuthal phase velocity of $100-200 \mathrm{~km} / \mathrm{s}$ of the TCS mode. While it is reasonable to conjure that the observed duskward expansion of the Pil wave sources is partly attributed to the azimuthal propagation of the TCS mode in our theoretical context, a direct comparison between them is unjustifiable from the methodology. Nevertheless, the inferred azimuthal propagation speed of the CD or the TCS mode are higher than that of the dipolarization at the geosynchronous orbit ( $\sim 70 \mathrm{~km} / \mathrm{s}$, see Liou et al., 2002). Their speed difference leads to the prediction that for GOES satellites far away from the onset sector the appearance of the Pi1 wave may also precede by a couple of minutes the start of the local dipolarization (Lessard et al., 2006), a timing sequence similar to what we obtained above from the tailward propagation difference of the CD front and the Pil waves. The coincidence again necessitates the use of global auroral images to help distinguish the azimuthally and radially propagational effects in properly interpreting the in-situ observations (e.g., Donovan et al., 2008).

When the local CD takes place, the wave activity exhibits an overall intensification and goes up to the higher frequency range $>0.1 \mathrm{~Hz}$. Figure 8 shows that the waves were also enhanced at frequency range well above the ion gyrofrequency. However, waves at those higher frequency band is tied exclusively to the excitation of local $\mathrm{CD}$, implying that they are only local process with little tailward propagation; otherwise one would expect to observe the waves at times other than the local $\mathrm{CD}$, as a remotely propagated effect from the onset origin, similar to the $\sim 20$ s period waves we analyze above. The overall spectrum becomes rather broadband during the local $\mathrm{CD}$, suggesting the possibility of plasma instabilities with different spatiotemporal characteristic as well as distinct frequency bands, e.g., ballooning (mainly Pi2), the TCS mode (Pi1), and the CCI (above and possibly overlap with the upper edge of the Pi1). During this most active period of substorms there are also other viable mechanisms generating Pi1/Pi2 waves (e.g., Holter et al., 1995; Volwerk et al., 2008). Those instability/wave processes may coexist and cooperate, driving the current sheet into a turbulence state (Lui, 2002; Cheng, 2004).

\section{Conclusions}

In this study we investigated the ULF wave activities, mainly in the Pi1 and Pi2 bands, in the crucial interval from minutes preceding the substorm EP onset to minutes after the local CD. The observed waves were pieced together into a synthetic scenario of various plasma wave modes during the substorm evolution. Main results from this study include,
1. We confirmed the result of Saito et al. (2008b) on the emergence of Pi2 band waves 1-2 min prior to the substorm EP onset. Such waves were tentatively attributed to the ballooning mode that may act as the seed perturbation leading to subsequent activities including the $\mathrm{EP}$ onset. In our scenario the ballooning mode interacts with the local current sheet and aids in its extreme thinning in final minutes before the local $\mathrm{CD}$, with some salient symptoms such as the ion temperature drop at the current sheet boundary (Liang et al., 2009) and deviations from the frozen-in flux condition (i.e., ion demagnetization). The interaction makes the local current sheet more prone to disruption.

2. The azimuthal wavelength estimated from the Dopplershifted ballooning mode and that inferred from ground ASI observation matched each other, consistent with our proposal that the longitudinal wave-like structuring during the initial auroral breakup reported by Liang et al. (2008) might be a manifestation of the ballooning mode.

3. Pi1 band (10-30s) waves emerging shortly after the EP onset were attributed to the propagation effect of the TCS mode investigated by Liu and Liang (2009).

4. The Pi1 waves characteristic of the above TCS mode were usually observed minutes prior to the local CD onset, implying that their tailward propagation speed is much faster than the $\mathrm{CD}$ front.

5. During the local $\mathrm{CD}$, broadband wave activities spanned from the Pi2 band to well above the ion-gyrofrequency. The spectrum suggests the coexistence of various plasma instabilities with different frequency characteristics, e.g., ballooning, TCS mode, and the CCI, during the local CD.

Acknowledgements. THEMIS was developed under the NASA Explorer Program. Funding for its GBO operation and data retrieval was provided by the CSA. We acknowledge the whole THEMIS team for their excellent work on this great mission. This study is supported by CSA and NSERC.

Topical Editor R. Nakamura thanks two anonymous referees for their help in evaluating this paper.

\section{References}

Arnoldy, R., Posch, J., Engebretson, M., Fukunishi, H., and Singer, H.: Pi1 magnetic pulsations in space and at high latitudes on the ground, J. Geophys. Res., 103(A10), 23581-23591, 1998.

Asano, Y., Mukai, T., Hoshino, M., Saito, Y., Hayakawa, H., and Nagai, T.: Evolution of the thin current sheet in a substorm observed by Geotail, J. Geophys. Res., 108(A5), 1189, doi:10.1029/2002JA009785, 2003.

Auster, H. U., Glassmeier, K. H., Magnes, W., et al.: The THEMIS fluxgate magnetometer, Space Sci. Rev., 141, 235-264, doi:10.1007/s11214-008-9365-9, 2008. 
Cheng, C. Z.: Physics of substorm growth phase, onset, and dipolarization, Space Sci. Rev., 113, 207-270, 2004.

Donovan, E., Liu, W., Liang, J., Spanswick, E., et al.: Simultaneous THEMIS in situ and auroral observations of a small substorm, Geophys. Res. Lett., 35, L17S18, doi:10.1029/2008GL033794, 2008.

Erickson, G. M., Maynard, N. C., Burke, W. J., Wilson, G. R., and Heinemann, M. A.: Electromagnetics of substorm onsets in the near-geosynchronous plasma sheet, J. Geophys. Res., 105, 25265-25290, 2000.

Holter, Ø., Altman, C., Roux, A., et al.: Characterization of low frequency oscillations at substorm breakup, J. Geophys. Res., 100(A10), 19109-19119, 1995.

Lessard, M. R., Lund, E. J., Jones, S. L., Arnoldy, R. L., Posch, J. L., Engebretson, M. J., and Hayashi, K.: Nature of Pi1B pulsations as inferred from ground and satellite observations, Geophys. Res. Lett., 33, L14108, doi:10.1029/2006GL026411, 2006.

Liang, J., Donovan, E. F., Liu, W. W., Jackel, B., Syrjäsuo, M., Mende, S. B., Frey, H. U., Angelopoulos, V., and Connors, M.: Intensification of preexisting auroral arc at substorm expansion phase onset: Wave-like disruption during the first tens of seconds, Geophys. Res. Lett., 35, L17S19, doi:10.1029/2008GL033666, 2008.

Liang, J., Liu, W. W., and Donovan, E. F.: Ion temperature drop and quasi-electrostatic electric field at the current sheet boundary minutes prior to the local current disruption, J. Geophys. Res., in review, 2009.

Liou, K., Meng, C.-I., Lui, A. T. Y., Newell, P. T., and Wing, S.: Magnetic dipolarization with substorm expansion onset, J. Geophys. Res., 107(A7), 1131, doi:10.1029/2001JA000179, 2002.

Liu, W. W.: Physics of the explosive growth phase: Ballooning instability revisited, J. Geophys. Res., 102, 4927-4931, 1997.

Liu, W. W., Liang, J., and Donovan, E. F.: Interaction between kinetic ballooning mode and thin current sheet: Quasielectrostatic field, local onset, and global characteristics, Geophys. Res. Lett., 35, 35, L20107, doi:10.1029/2008GL035757, 2008.

Liu, W. W. and Liang, J.: Disruption of magnetospheric current sheet by quasi-electrostatic field, Ann. Geophys., 27, 1941-1950, 2009, http://www.ann-geophys.net/27/1941/2009/.

Lui, A. T. Y., Chang, C.-L., Mankofsky, A., Wong, H.-K., and Winske, D.: A Cross-Field Current Instability for Substorm Expansions, J. Geophys. Res., 96(A7), 11389-11401, 1991.

Lui, A. T. Y.: A synthesis of magnetospheric substorm models, J. Geophys. Res. 96, 1849-1856, 1991.

Lui, A. T. Y. and Najmi, A.-H.: Time-frequency decomposition of signals in a current disruption event, Geophys. Res. Lett., 24, 3157-3160, 1997.

Lui, A. T. Y.: Multiscale phenomena in the near-Earth magnetosphere, J. Atmos. Solar-Terr. Phys., 64, 125-143, 2002.

Lui, A. T. Y., Yoon, P. H., Mok, C., and Ryu, C.-M.: Inverse cascade feature in current disruption, J. Geophys. Res. 113, A00C06, doi:10.1029/2008JA013521, 2008.

McFadden, J. P., Carlson, C. W., Larson, D., et al.: The THEMIS ESA plasma instrument and in-flight calibration, Space Sci. Rev., 141, 277-302, doi:10.1107/s11214-008-9440-2, 2008.
Mende, S. B., Harris, S. E., Frey, H. U., et al.: The THEMIS array of ground-based observatories for the study of auroral substorms, Space Sci. Rev., 141, 357-387, doi:10.1007/s11214-008-9380, 2008.

Murphree, J., Johnson, M., Cogger, L., and Hearn, D.: Freja UV Imager observations of spatially periodic suroral distortions, Geophys. Res. Lett., 21(17), 1887-1890, 1994.

Ohtani, S.: On the tailward expansion of current disruption during substorms, Adv. Space. Res., 13, 265-268, 1993.

$\mathrm{Pu}, \mathrm{Z}$. Y., Korth, A., Chen, Z. X., et al.: MHD drift ballooning instability near the inner edge of the near-Earth plasma sheet and its application to substorm onset, J. Geophys. Res., 102(A7), 14397-14406, 1997.

Roux, A., Le Contel, O., Coillot, C., et al.: The search coil magnetometer for THEMIS, Space Sci. Rev., 141, 265-275, doi:10.1007/s11214-008-9455-8, 2008.

Saito, M. H., Miyashita, Y., Fujimoto, M., Shinohara, I., Saito, Y., and Mukai, T.: Modes and characteristics of low-frequency MHD waves in the near-Earth magnetotail prior to dipolarization: Fitting method, J. Geophys. Res., 113, A06201, doi:10.1029/2007JA01277, 2008a.

Saito, M. H., Miyashita, Y., Fujimoto, M., Shinohara, I., Saito, Y., Liou, K., and Mukai, T.: Ballooning mode waves prior to substorm-associated dipolarizations: Geotail observations, Geophys. Res. Lett., 35, L07103, doi:10.1029/2008GL033269, 2008b.

Savitzky, A. and Golay, M. J. E.: Smoothing and Differentiation of Data by Simplified Least Squares Procedures, Analyt. Chem., 36, 1627-1639, doi:10.1021/ac60214a047, 1964.

Shiokawa, K., Shinohara, I., Mukai, T., Hayakawa, H., and Cheng, C. Z.: Magnetic field fluctuations during substorm-associated dipolarizations in the nightside plasma sheet around $X=-10 R_{E}$, J. Geophys. Res., 110, A05212, doi:10.1029/2004JA010378, 2005.

Sigsbee, K., Cattell, C. A., Fairfield, D., Tsuruda, K., and Kokubun, S.: Geotail observations of low-frequency waves and highspeed earthward flows during substorm onsets in the near magnetotail from 10 to $13 R_{E}$, J. Geophys. Res., 107(A7), 1141, doi:10.1029/2001JA000166, 2002.

Trondsen, T. and Cogger, L.: A survey of small-scale spatially periodic distortions of auroral forms, J. Geophys. Res., 103(A5), 9405-9415, 1998.

Volwerk, M.: Multi-satellite observations of ULF waves, Magnetospheric ULF waves: synthesis and new directions, p. 109, edited by: Takahashi, K., Chi, P. J., Denton, R. E., and Lysak, R. L., AGU Monograph, 169, 2006.

Volwerk, M., Nakamura, R., Baumjohann, W., Uozumi, T., Yumoto, K., Klecker, B., Balogh, A., and Reme, H.: Pi2 Observations by Cluster, ICS-9, Graz, 2008.

Voronkov, I., Donovan, E. F., Jackel, B. J., and Samson, J. C.: Large-scale vortex dynamics in the evening and midnight auroral zone: Observations and simulations, J. Geophys. Res., 105(A8), 18505-18518, 2000.

Voronkov, I., Rankin, R., Frycz, P., Tikhonchuk, V., and Samson, J.: Coupling of shear flow and pressure gradient instabilities, J. Geophys. Res., 102(A5), 9639-9650, 1997. 